\title{
Hepcidin and Ferritin Levels in Fever of Unknown Origin: Is There a New Biomarker?
}

\author{
Ana López-Aparicio ${ }_{1}^{1}$ Alejandro García-Espona-Pancorbo ${ }^{1}$, Marta Clavero-Olmos ${ }^{1}$, \\ Inmaculada Muñoz-Roldán ${ }^{1}$, Alejandro del Castillo-Rueda ${ }^{2}$ \\ ${ }^{1}$ Servicio Medicina Interna, Hospital General Universitario Gregorio Marañón, Madrid, Spain \\ 2Unidad de Ferropatología y Radicalosis, Servicio de Medicina Interna, Hospital General Universitario Gregorio Marañón, Madrid, Spain.
}

Received: 02/06/2015

Accepted: $23 / 08 / 2015$

Published: $21 / 09 / 2015$

\begin{abstract}
How to cite this article: Lopez-Aparicio A, García-Espona-Pancorbo A, Clavero-Olmos M, Muñoz-Roldán I, del Castillo-Rueda A. Hepcidin and ferritin levels
\end{abstract} in fever of unknown origin: is there a new biomarker?. EJCRIM 2015;2:doi:10.12890/2015_000240

Conflicts of Interests: The authors declare that there are no competing interests.

Patient's Consent: The authors declare they obtained patient's consent for publication.

This article is licensed under a Commons Attribution Non-Commercial 4.0 License

\section{ABSTRACT}

Background and objectives: Significantly elevated serum ferritin levels are associated with both iron overload and some inflammatory conditions. Hepcidin is a protein that interferes with iron absorption in inflammatory states and acts as an acute-phase reactant.

Materials and methods: Here we report the case a 33-year-old patient who presented with high fever, skin lesions and arthralgia lasting for 2 weeks. His ferritin level was 13,800 $\mathrm{\mu g} / \mathrm{l}$ and his hepcidin level was $61 \mathrm{ng} / \mathrm{dl}$.

Results: The final diagnosis was adult onset Still's disease. The condition evolved satisfactorily with steroid treatment, but after several weeks the patient presented with an unexpected recurrence.

Conclusions: Hepcidin is a good inflammatory marker that could be useful in the differential diagnosis of hyperferritinaemia.

\section{LEARNING POINTS}

- There are no specific markers for adult onset Still's disease, but hyperferritinaemia is a characteristic laboratory finding.

- Hepcidin, as an inflammatory marker, might be useful in the diagnosis of this disorder.

- Some factors, such as infections or vaccination, could cause recurrence when steroid doses are being tapered.

\section{KEYWORDS}

Still's disease, hyperferritinemia, hepcidin.

\section{INTRODUCTION}

Adult onset Still's disease (AOSD) is an inflammatory disorder characterized by fever, arthralgia and an evanescent rash ${ }^{[1]}$. Hyperferritinaemia is one of the most characteristic laboratory findings ${ }^{[2]}$. Recurrences have been related to treatment disruption or improper tapering of steroid doses. However, very few cases of AOSD recurrence due to influenza vaccination have been reported.

\section{CASE REPORT}

A 33-year-old man was admitted to the emergency department (ED) complaining of skin lesions, fever and migratory arthralgia lasting for 2 weeks. He had no previous illnesses and was not taking any medication. He was a prisoner in a penitentiary.

Physical examination showed high fever and pruritic erythematous macula on the trunk and extremities and worsened by scratching (Fig. 1). 
No other remarkable findings on physical examination were found. Laboratory tests revealed normocytic and normochromic anaemia, leukocytosis with neutrophilia, mild hypertransaminasemia and a remarkable acute-phase reactant elevation (PCR 30.8 mg/dl, procalcitonin $2.08 \mu \mathrm{g} / \mathrm{l})$. Urine investigation and chest radiography conducted in the ED did not show any pathological findings.

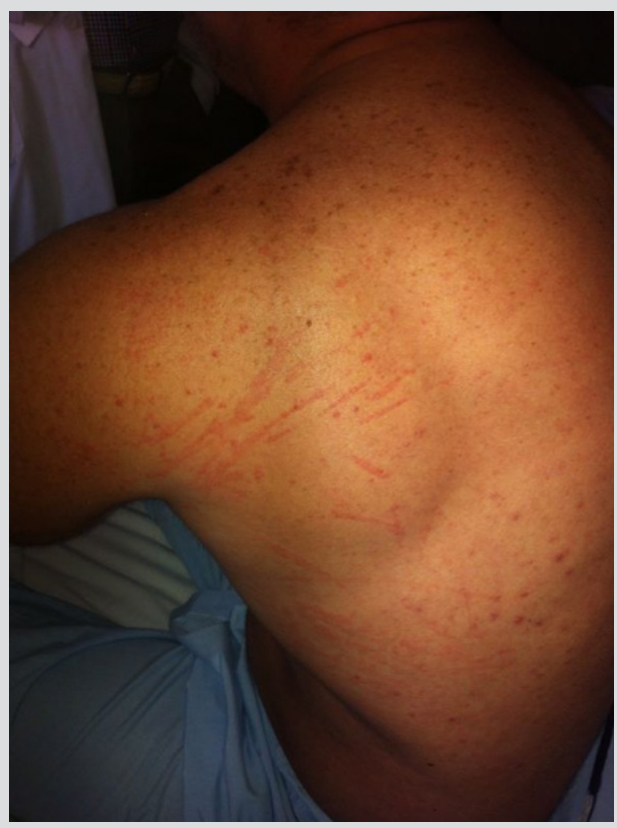

Figure 1: Erythematous maculopapular rash on the trunk and extremities (evanescent rash) worsened by scratching (Koebner phenomenon).

The patient's rash worsened and he was very ill during his first days in hospital due to persistent high fever which kept him bed-ridden. Additional tests confirmed the abnormalities first observed, and revealed a serum ferritin level of $13,800 \mu \mathrm{g} / \mathrm{l}$. All immunity markers as well as blood cultures and serological tests were negative (HIV, Ebstein-Barr virus, syphilis, hepatotropic viruses, cytomegalovirus, B19 parvovirus, Leishmania). We determined serum hepcidin concentrations using a commercial enzyme-linked immunosorbent assay kit (DRG Instruments, Marburg, Germany) ${ }^{[3]}$ that demonstrated a level of $61 \mathrm{ng} / \mathrm{dl}$ (normal range 2-20 ng/dl). Complete body computed tomography showed multiple intrathoracic, mediastinal and intra-abdominal nodes with splenomegaly and mild bilateral pleural effusion. A skin biopsy was taken.

Due to the clinical suspicion, treatment with steroids was initiated. The initial dose of prednisone $30 \mathrm{mg}$ per day had an excellent response. The skin biopsy was compatible with AOSD (Fig. 2). The patient was asymptomatic when he was discharged from hospital on a slowly tapering dose of steroids.
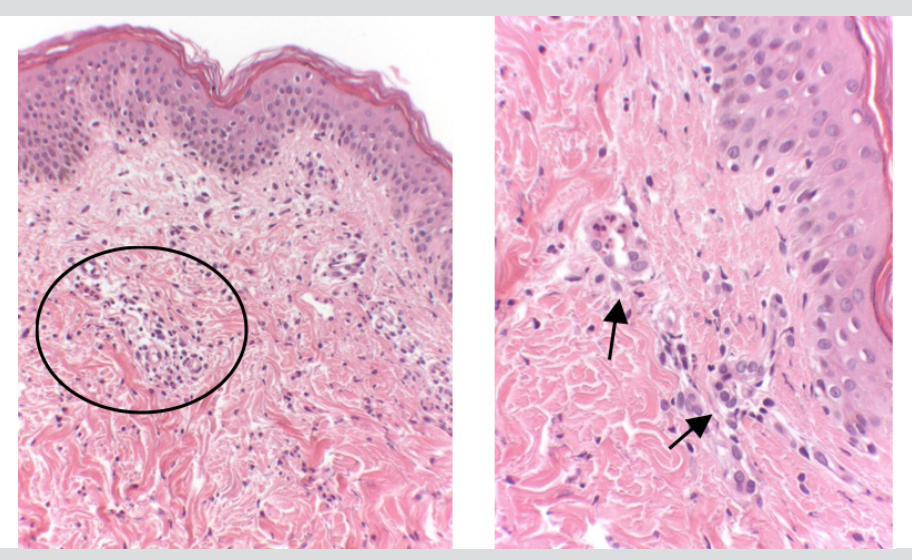

Figure 2: Cutaneous histopathology revealed mild epidermal oedema and a perivascular acute inflammatory infiltrate (mostly neutrophils) in conserved dermis; although these are non-specific findings, they are suggestive of adult onset Still's disease. 
Six weeks after hospital discharge, the patient represented with a recurrence of his Still's disease (fever, skin rash and arthralgia). It is of note that the patient was taking a very low dose of steroids, and had also received the influenza vaccine 4 days before the new symptoms started. Acute-phase reactants were elevated (fibrinogen $>1,000 \mathrm{mg} / \mathrm{dl}$, PCR 30.4) and ferritin level was $1454 \mu \mathrm{g} / \mathrm{l}$. Serology revealed the same results as previously. Initial therapy with oral steroids (prednisone $60 \mathrm{mg}$ per day) produced no response. An intravenous steroid bolus (methylprednisolone $250 \mathrm{mg}$ for 3 days) was administered twice with poor response. Due to the steroid resistance, immunomodulator therapy was initiated with methotrexate $20 \mathrm{mg}$ (plus oral steroids) but symptoms persisted. A decision to initiate tocilizumab $8 \mathrm{mg} / \mathrm{kg}$ was made. After $72 \mathrm{~h}$, symptoms had disappeared and blood tests had returned to normal. A few days after becoming asymptomatic the patient was discharged from hospital. His condition showed favourable evolution in the following check-ups.

\section{DISCUSSION}

Serum ferritin levels reflect both an acute-phase response and an inflammatory state in the organism. Pro-inflammatory cytokines regulate ferritin secretion. It is believed that very high ferritin levels may have a specific pathogenic role in some diseases, so some authors refer to a "hyperferritinaemic syndrome" found in AOSD, macrophage activation syndrome, septic shock and catastrophic antiphospholipid syndrome ${ }^{[1]}$. Hepcidin is a hormone synthesized in the liver and controls iron homeostasis. Its antimicrobial activity is due to the fact that it eliminates plasma iron so that intestinal absorption is avoided and iron is deposited in macrophages. This protein acts in inflammatory states and in anaemia of chronic inflammation by blocking ferroportin (the sole known cellular iron exporter and the hepcidin receptor), resulting in decreased iron absorption in enterocytes and preventing the release of iron from stores ${ }^{[4]}$. Hepcidin synthesis decreases in anaemia, hypoxia and haemochromatosis with iron overload, and increases in inflammatory disorders causing inflammatory anaemia. Hepcidin plays an important role in the differential diagnosis in hyperferritinaemic illnesses that present with anaemia or other unspecific symptoms. As there are no standardized laboratory tests that correlate clinical features with hepcidin levels, we analyzed our patient's hepcidin levels to check for any association with the diagnosis of AOSD ${ }^{[5]}$.

There is no evidence that hepcidin discriminates infectious from non-infectious fevers of unknown origin (FUOs). However, it plays an important role in patients with hyperferritinaemia by discriminating high ferritin levels due to an inflammatory state from those caused by iron overload.

We decided to measure hepcidin levels in our patient because of his extremely high ferritin levels (13,800 $\mu$ g/l); hepcidin is currently a biomarker of interest in the medical community and studies on it are periodically published in the medical literature.

Our group is especially interested in the role of hepcidin in inflammatory states, its potential role in these pathologies and its antimicrobial function. Funded by a medical scholarship, we therefore measure hepcidin levels in patients with hyperferritinaemia to study its behaviour in different pathologies. We conclude that hepcidin interferes with iron absorption and also acts as an acute-phase reactant. This new biomarker may in future be routinely measured in daily clinical practice to discriminate infectious from non-infectious FUOs.

Regarding AOSD recurrence, very few cases related to influenza vaccination have been described in the scientific literature. In our patient, two factors (low steroid dose and influenza vaccination) were probably responsible for the recurrence of AOSD, although we cannot reliably demonstrate the role of the vaccine.

\section{REFERENCES}

1. Kadavath S, Efthimion P. Adult-onset Still's disease-pathogenesis, clinical manifestations and new treatment options. Ann Med 2015;47:6-14.

2. Moore C, Ormseth M, Fuchs H. Causes and significance of markedly elevated serum ferritin levels in an academic medical centre. J Clin Rheum 2013;19:324-248.

3. Del Castillo-Rueda A, Mastkiv N, Moreno- Carralero MI, Morán-Jiménez MJ. Serum hepcidin levels in hospitalized patients. A pilot study. Am J Hematol 2013;88:E226.

4. Pietrangelo A. Hepcidin in human iron disorders: therapeutic implications. J Hepatol 2011;54:173-181.

5. Mecklenburg I, Reznik D, Fasler-Kan E, Drewe J, Belglinger C, Hruz P. Serum hepcidin concentrations correlate with ferritin in patients with inflammatory bowel disease. J Crohns Colitis 2014;8:392-397. 